\title{
Las técnicas constructivas en las murallas medievales de Almería
}

\author{
Building techniques in the medieval walls of Almería
}

\section{Pedro Gurriarán Daza}

Yamur S.L., Málaga, Spain, modromore@yahoo.es

\begin{abstract}
Almería was one of the most important cities in al-Andalus, a circumstance that was possible thanks to the strength of its port. Its foundation as an urban entity during the Caliphate of Córdoba originated a typical scheme of an Islamic city organized by a medina and a citadel, both walled. Subsequent city's growths, due to the creation of two large suburbs commencing in the eleventh century, also received defensive works, creating a system of fortifications that was destined to defend the place during the rest of the Middle Ages. In this work we will analyse the construction techniques used in these military works, which cover a wide period from the beginning of the tenth century until the end of the fifteenth century. Although ashlar stone was used in the Caliphate fortification, in most of these constructions bricklayer techniques were used, more modest but very useful. In this way, the masonry and rammed earth technique were predominant, giving rise to innumerable constructive phases that in recent times are being studied with archaeological methodology, thus to know better their evolution and main characteristics.
\end{abstract}

Keywords: Ashlar stone, rammed earth, masonry, Almería.

\section{Introducción}

El estudio de las técnicas constructivas en alAndalus ha sufrido una notable evolución en las últimas décadas, como consecuencia, principalmente, del desarrollo de la arqueología medieval y de nuevos métodos de análisis vinculados a la disciplina conocida como Arqueología de la Arquitectura. En muchos casos relacionada con trabajos de conservación, la renovada visión aportada por los estudios estratigráficos de edificios ha supuesto un salto cualitativo en el conocimiento que tenemos sobre la forma de construir y cómo se plasma en determinados sistemas edilicios. Los postulados de la Arqueología de la Arquitectura no solo definen las tipologías existentes, sino también los procesos evolutivos dentro de unos medios de producción de la arquitectura que se dan en cada fase. Estos sobrepasan al edificio y debe considerar, por ejemplo, los puntos de suministro de materiales, el transporte, así como los actores intervinientes, desde el promotor hasta los especialistas incluidos en posibles talleres. Quizás son las fortificaciones el tipo de construcción que más se han prestado a este tipo de análisis en los últimos años, y donde poco a poco se han ido consiguiendo mayores avances, ajustando muchas cronologías.

Dentro de este panorama general, Almería se configura como un caso especial y susceptible de poder obtener un caudal de datos ciertamente relevante y resaltar las defensas medievales de este enclave dentro del conjunto de la arquitectu- 
ra andalusí. En primer lugar, se identifican actuaciones que abarcan desde periodo altomedieval hasta la toma de la plaza por los Reyes Católicos a finales del siglo XV. Por otra parte, y a pesar de las destrucciones acaecidas desde mediados del siglo XIX, se conservan amplios tramos de la cerca urbana incluyendo la alcazaba, lo que, unido a la información planimétrica y fotográfica de las partes desaparecidas, configuran un material de estudio destacado. Además, muchas de las estructuras no han sufrido agresivas restauraciones, lo que repercute en una adecuada interpretación. Como consecuencia, se ha ido creando un importante corpus documental, que va arrojando luz sobre un yacimiento complejo, en el que coyunturas especiales como asedios o terremotos dejaron su impronta en los muros.

En las próximas líneas se definirán los avances obtenidos últimamente en el estudio de las técnicas constructivas de las fortificaciones medievales de Almería, centrando el discurso en las tipologías más significativas. Se estudiará la información obtenida sobre todo en lecturas estratigráficas de paramentos, así como las observaciones que el autor ha podido realizar como director de varios de proyectos de restauración en la alcazaba. Como se expondrá a continuación, aunque las certezas son cada vez mayores, aún existen dudas, especialmente sobre las defensas tempranas del enclave.

\section{Del puerto de Pechina a la ciudad nazarí. Contexto histórico de las defensas almerienses}

Si bien Almería desde una perspectiva urbana se ha de entender como una fundación andalusí (madīna muhdata) (Lirola, 2005, p. 19), la arqueología ha venido a demostrar que en su solar existía algún tipo de asentamiento en periodo antiguo, localizado en el espacio comprendido entre la peña rocosa de la alcazaba y el terreno llano que baja hacia el litoral donde después creció la madina (Pardo, 2012, pp. 562-563). El núcleo de Pechina (Baŷŷāna), situado varios kilómetros tierra adentro en la ribera del Andarax, sería la principal ciudad de toda la zona durante el emirato. Su historia y desarrollo no se entiende sin su relación con el mar y con su puerto (Marsā o Furdat Baŷȳanna), donde luego crecería Almería. Según las fuentes, allí se establecieron en ribāt contingentes yemeníes a mediados del siglo IX y erigieron las primeras obras defensivas. En concreto, al-Himyarī refiere que "los normandos habían avanzado hasta Almería y habiendo dado la vuelta a las costas de al-Andalus y del litoral africano, los árabes utilizaron Almería como observatorio (mira'ā) y construyeron allí torres de guardia (mahāris) [...]" (Al-Himyarī, 1938, 47). Manuel Acién reflexionó sobre el término mahāris y concluyó que habrían de interpretarse como recintos defensivos que alojaban una pequeña guarnición, consagrada al ribāt (Acién, 2005, 77-78). La arqueología ha confirmado que, al menos uno de ellos, se puede localizar sobre la peña de la alcazaba.

Baŷyāna creció y se consolidó al amparo de una intensa actividad portuaria tras el establecimiento, en 884 , de un conjunto de marinos (albahrīyyūn). En 922-923 Pechina y su territorio fueron anexionados por el Estado omeya, quien convirtió su puerto en la base principal de la flota. Otra fecha clave que condicionaría el devenir de Almería fue 955, cuando una escuadra fatimí asaltó el puerto y durante varios días se dedicó al pillaje y la destrucción. La reacción de Abd al-Raḥmān III fue contundente y decidió refundar el enclave con el rango de madīna. Esta decisión no solo cambiaba su estatus, sino que implicaba la construcción de la mezquita mayor, la alcazaba, las murallas urbanas y, seguramente, la renovación del arsenal, dentro de un esquema arquetípico de ciudad islámica.

De este modo, a finales del califato quedaba constituida la estructura urbana básica de Almería en torno a la doble polaridad entre el recinto de la madīna, bañado por el mar al sur, y la fortaleza de la alcazaba, alzada sobre el cerro inmediato justo al norte. La posterior capital taifa creció con dos grandes arrabales amurallados, situado el más rico y pujante hacia occidente del núcleo primigenio (al-Hawd), mientras que el otro de mayor extensión se fundó a oriente (al-Mușallā). La preponderancia comercial y económica de Almería, sostenida por su importancia portuaria, se acentuó desde entonces y alcanzó su cénit en periodo almorávide. Algunos 
autores nos aportan datos sobre la realización de obras defensivas a comienzos del siglo XII, como al-Rusati, quien refiere cómo en sus murallas "lo deteriorado se unía a lo nuevo" (Lirola Delgado, 2005, p. 51). En el al-Bayān al-Mugrib se habla de un impuesto con el que se costeó la fortificación de Córdoba, Sevilla, Granada y Almería en 1125-1126; en concreto, al citar a esta última ciudad se dice que "se encargó de mirar por las murallas de Almería un hombre de ella [...] y se acabó la muralla, según lo exigía de fortaleza y hermosura, con el menor gasto, sin golpe ni cárcel” (Ibn 'Iḍārī, 1963, p. 170).

La suerte de Almería cambió de forma radical tras el decenio de ocupación cristiana entre 1147 y 1157, entrando en lo que algún autor ha llamado una sucesión de hitos desgraciados o "quebrantos" (Molina López, 2005, p. 19). Aunque existe un fenómeno de regresión urbana, la plaza seguiría en buen estado de defensa, lo que justifica las dificultades que sufrieron los almohades durante los dos intentos de recuperación, concluidos en 1157 tras un duro sitio. Tras este acontecimiento, la arqueología ha comprobado cómo la actividad edilicia se concentró en reforzar las defensas existentes, situación que se habría de repetir en periodo nazarí, ya que la ciudad no habría de crecer más.

\section{La construcción de las murallas medievales de Almería. Principales tipologías}

Si bien Almería es un lugar rodeado de afloramientos de un tipo de piedra muy adecuada para la construcción, como vemos en las numerosas canteras históricas de calcarenita que pueblan sus laderas, la piedra tallada o sillería nunca fue la técnica predominante en sus murallas andalusíes. En general, destacan técnicas que se pueden denominar de albañil, en las que la especialización del encargado de ejecutar las obras y el número de operaciones totales para concluir un muro es mucho menor que en el caso de la cantería. En esa categoría se incluirían mamposterías y sillarejos, así como la técnica del tapial, que será la protagonista sin discusión a partir del siglo XI (Gurriarán, 2014, p. 277). No se trataría de una situación exclusivamente local, sino que respondería a un fenómeno generalizado en alAndalus.

No es la intención de este trabajo hacer un estudio pormenorizado de todas las fábricas existentes, ya que sobrepasaría sus condiciones de partida. De este modo solo se expondrán las técnicas edilicias más representativas, indicando sus características principales y aquellos datos arqueológicos disponibles que sirven para definirlas tanto técnica como cronológicamente.

\subsection{Materiales pétreos}

\subsubsection{Mampostería careada irregular}

La existencia de mamposterías simples e irregulares, entendidas como el tipo más básico de este tipo de fábrica, es bastante común en las fortificaciones almerienses, especialmente en la alcazaba. Se trata de una solución utilitaria que requiere de poca especialización por sus constructores, por lo que era habitual su empleo para realizar reparaciones y obras de urgencia en diversos periodos. Sin embargo, centra nuestra atención aquí unas fábricas que han sido estudiadas en la alcazaba, datadas por la arqueología como anteriores a periodo califal.

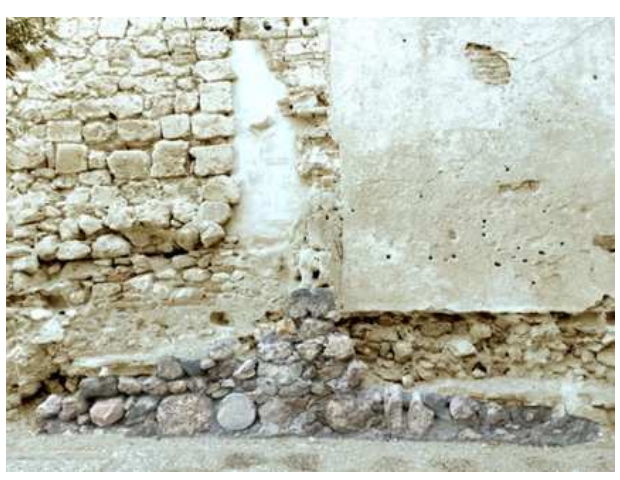

Fig. 1. Fábricas precalifales en la base del Muro de la Vela de la alcazaba, resaltadas en gris (P. Gurriarán).

En efecto, en las exploraciones arqueológicas efectuadas en el segundo recinto de esta ciudadela se pudieron identificar una serie de potentes estructuras debajo de las cimentaciones de los palacios andalusíes, interpretadas como la más antigua fase defensiva de esta fortificación (Arias de Haro, Alcalá Lirio, 2011, pp. 129- 
130). Estructuras defensivas coetáneas se estudiaron junto al Baño de la Tropa (Arias de Haro, Alcalá Lirio, 2011, pp. 71-72), así como en el Muro de la Vela.

Llama la atención la técnica constructiva empleada en su construcción, ya que, al predominio de la mampostería careada e irregular, extraída en el propio lugar y tomada con mortero de cal, se une la presencia de alguna pieza de acarreo como vemos con un fuste en el Muro de la Vela, siguiendo una práctica muy común en la arquitectura altomedieval (Fig. 1). Por último, estas estructuras protegían su superficie mediante un enlucido de base caliza.

Desde luego, se trata de una técnica constructiva modesta y típica de la albañilería, muy habitual en numerosos husșūn estudiados en la costa andaluza durante el emirato (El Nicio, Bezmiliana, Jate, etc.) (Navarro, et al., 1998). Es sugerente pensar que se trata de uno de los mahāris referidos en esa época, que seguramente seguiría en funcionamiento en la primera mitad del siglo $\mathrm{X}$.

\subsubsection{Sillería escuadrada}

La intervención constructiva de las autoridades cordobesas a partir de 955 hubo de ser de gran calado, ya que implicaba una serie de obras emblemáticas entre las que sobresalían las murallas; encajaba en el concepto de una actuación prestigiosa y representativa del poder que la emanaba. Las referencias textuales hablan de la construcción de la muralla por 'Abd al-Raḥmān III, resaltando el empleo de la piedra, como sucede en otras actuaciones coetáneas como Ceuta o Tortosa. Esa información encajaría con la costumbre de emplear canteros en sus proyectos emblemáticos, sin embargo, el problema existente en Almería es que hay pocos datos arqueológicos que refrenden lo dicho por las fuentes. De la "muralla inexpugnable de piedra que construyó al-Nāșir" o la "prodigiosa muralla" que cercaba la madina antigua, como exaltó al-'Ud̆rì (Lirola, 2005, p. 31), queda poco rastro y se reduce a zonas dispersas.

La organización del trabajo de la piedra en la urbe califal rotaba alrededor del aprovechamiento de un conjunto de canteras localizadas junto a la ciudad, sobre todo en la zona occidental, en torno al actual barrio de La Chanca (Cara, Alonso, Berbel, 2006). La obra mejor conservada de sillería escuadrada aparejado a soga y tizón se alza en el interior de la actual iglesia de San Juan Evangelista y formaría parte del muro de la qibla de la desaparecida mezquita mayor. Se construye mediante sillares de arenisca de buena labra, aparejados sistemáticamente mediante una soga y dos y tres tizones como norma general. Las juntas son muy finas y se rellenan mediante mortero de cal de gran blancura. Por lo que respecta a restos de tipo defensivo, aparecen en la alcazaba, siempre en el segundo recinto y aparentemente reformando las mamposterías que describíamos en el apartado anterior. Se localizan formando parte del Muro de la Vela y junto al Baño de la Tropa (Gilotte, et al., 2010, p. 225) (Fig. 2), donde se aparejan a soga y tizón, pero en hiladas alternas. El trabajo de estos especialistas se estudia también en otras construcciones de la ciudadela, como sucede con las bóvedas de medio cañón del aljibe conocido como califal, e incluso en la doble puerta en recodo de la zona de los palacios, erigida hacia finales del siglo $\mathrm{X}$ e inicios del siguiente (Arias de Haro, Alcalá Lirio, 2011, pp. 130).

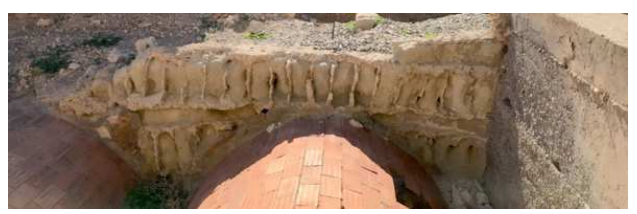

Fig. 2. Muro de soga y tizón califal en el segundo recinto de la alcazaba (P. Gurriarán).

En el paño conservado en el centro de interpretación "Puerta de Almería", aparecen también sillares de módulo califal, pero su puesta en obra con engatillados denota su reaprovechamiento, tal vez del propio arsenal del siglo X. Otros restos del frente marítimo fueron exhumados en un solar de la calle Chafarinas, en este caso, el relleno de sillares de la muralla, irregular y de pobre desbastado.

Por último, se conserva una cuidada estructura de soga y tizón en la cerca del cerro de San Cristóbal, en concreto en la fase original del postigo allí existente. Las piezas de soga y tizón 
se alternan en hiladas sucesivas en los laterales del pasadizo (Fig. 3), de modo similar a como veíamos en la alcazaba, y la pericia en la labra se observa también en las finas dovelas del dintel de cierre. El origen posterior al califato de esa obra implicaría que la labor de estos especialistas canteros se pudo prolongar durante décadas, organizados siempre mediante talleres.

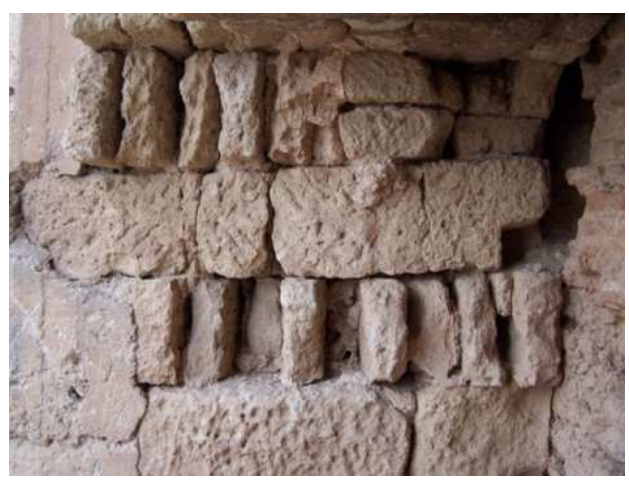

Fig. 3. Detalle del postigo conservado en la muralla del Cerro de San Cristóbal (P. Gurriarán).

\subsubsection{Mampostería careada regular}

Las mamposterías son comunes en las murallas almerienses, como ya se dijo, pero las que nos ocupan son ciertamente singulares por su especial puesta en obra y sus implicaciones arqueológicas e históricas. En efecto, llama la atención en la cortina que cierra el altozano de San Cristóbal la presencia de cuatro torres de piedra insertas entre las estructuras de tapial predominantes. Se trata de uno más de los elementos de distintas épocas que han servido para reforzar el punto más vulnerable de las defensas de Almería, sometido a distintos asedios a lo largo de toda la Edad Media.

Las torres se alzan con trazado semicircular, en algunos casos peraltado y su homogeneidad edilicia denota un mismo impulso constructor (Fig. 4). Son estructuras macizas que sobresalen por encima del nivel del adarve y apoyan en una zapata de grandes bloques de piedra caliza.

Las fábricas que forman el cuerpo principal se construyen con mampostería careada irregular de gran formato, dispuesta en hiladas, asegurando el ajuste y recalce final de las piezas median- te un enripiado que caracteriza el aparejo final. Hay que destacar que las piezas se toman con un mortero de cal que crea grandes rebabas, en ocasiones a modo de vitolas. La anchura de estas bandas de mortero es lo suficientemente grande como para resaltar sobre ellas unas cintas a modo de retícula, simulando un despiece de sillería. En una de las torres, este tratamiento epidérmico fue sustituido por un envitolado que incluye lágrimas esgrafiadas, además de fragmentos de cerámica vidriada. Aparecen otras fábricas complementarias y coetáneas a la obra descrita, como los aparejos con bandas de ladrillo y cajones mixtos de aparejo toledano de mampuesto y ladrillo que se localizan en el frente interior de una de las torres. El porqué de esta variación, siendo aparentemente sincrónica del resto de la construcción, es difícil de precisar, pero podría responder al trabajo de otras cuadrillas de albañiles, cada una de ellas especializadas en un tipo de construcción.

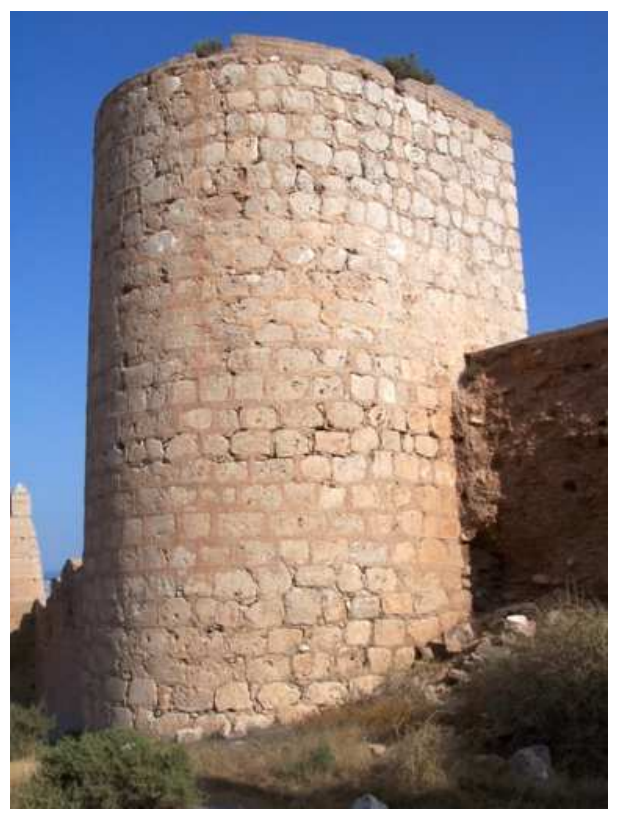

Fig. 4. Torre de mampostería en la muralla del Cerro de San Cristóbal (P. Gurriarán).

En un trabajo publicado hace unos años, ya reflexionamos sobre el posible origen de estas estructuras extrañas en la edilicia andalusí de Almería, y la conclusión a la que se llegó es que 
se trataba de torres erigidas en el decenio de ocupación cristiana a mediados del siglo XII (Gurriarán, Márquez, 2005, pp. 68-70).

\subsection{Materiales hormigonados}

\subsubsection{Tapias mixtas}

En diversos puntos de las fortificaciones de Almería se observan unas tapias cuya ejecución es singular y merecen que se analicen como obras mixtas, según reza el enunciado. En efecto, bajo la apariencia superficial de una tapia de tipo convencional se esconde un sistema que introduce dentro de los cajones, ocultos al exterior, una serie de pilares de piedra de ancho variable (60-70 $\mathrm{cm}$ aproximadamente) que apoyan a intervalos regulares sobre cadenas horizontales dispuestas en la base del cajón. La profundidad a la que se introducían estos elementos tampoco es regular, ya que existen casos en los que las piezas pétreas asoman y otros en los que permanecerían bien embutidas en el interior de la tapia (Fig. 5). La mezcla de relleno posee un árido de grano medio, aunque también se usan ripios y pequeños mampuestos en menor proporción. La cara exterior presenta un ligero calicostrado, mientras que su epidermis final es la resultante del desencofrado.

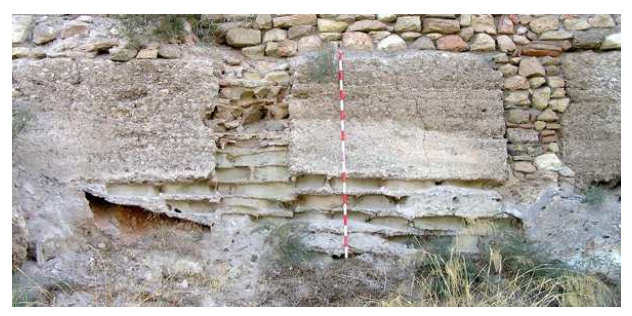

Fig. 5. Tapias mixtas en la ladera norte del segundo recinto de la alcazaba (P. Gurriarán).

Esta técnica constructiva se ha de entender como un sistema plenamente asentado en la zona, pues ya existen paralelos en la arquitectura doméstica de la vecina Bâŷyana, donde los métodos constructivos predominantes presentan un basamento de mampostería, sobre el que se alzan cajones de tierra delimitados por machones de sillares (Castillo, Martínez, 1990, p. 112). Este caso es relevante, al identificar una transferencia tecnológica desde una escala modesta a otra de tipo monumental, lo que a su vez habla bien claro de su versatilidad.

La presencia de estas singulares fábricas encofradas se repite en distintos puntos del perímetro amurallado de la alcazaba, generalmente al pie de las estructuras y en las partes más antiguas por simple relación estratigráfica. Aunque se desconoce su cronología absoluta, es posible que se ejecutaran entre finales del siglo X y comienzos del siguiente. En cualquier caso, esta forma de construir habría de proseguir en otras obras militares a caballo entre los siglos XI y XII, como demuestran algunos hallazgos arqueológicos realizados en la cerca del arrabal de alMuṣallā (Alcaraz, 2002, pp. 18-20).

\subsubsection{Tapias calicostradas de grano fino}

En las obras defensivas que aún permanecen en pie en San Cristóbal, La Hoya y en el barrio de La Chanca aparecen unas fábricas encofradas uniformes y muy bien construidas que son tipo de calicostrado. También se localizan aisladas en algunas zonas del frente norte del primer recinto de la alcazaba. Esta variedad de tapia se caracteriza por presentar una capa o costra exterior rica en mortero de cal, lo que le confiere gran resistencia, proporción de aglomerante que se hace más pobre en su núcleo hasta casi desaparecer y volverse casi terroso. En este caso, la mezcla es de grano fino y adopta un color ocre. La terminación final es tan cuidada que aún se observa generalmente la impronta de las cabezas de los clavos de los encofrados recorriendo la superficie de las tapias.

Estas tapias calicostradas sirven para erigir las torres de flanqueo tan uniformes que se levantan en las murallas de estos arrabales, lo que lleva a asegurar su construcción en un mismo impulso y la participación de una misma cuadrilla de tapiadores (Fig. 6). Las torres son macizas hasta el nivel del adarve de la muralla, desde donde se levantarían dos estancias superpuestas cerradas por forjados de madera. De forma sistemática, presentan una pareja de saeteras en su alzado frontal, mientras que en los laterales solo aparece uno de estos estrechos huecos. Las torres se coronan con merlones prismáticos rematados por simples albardillas piramidales. 


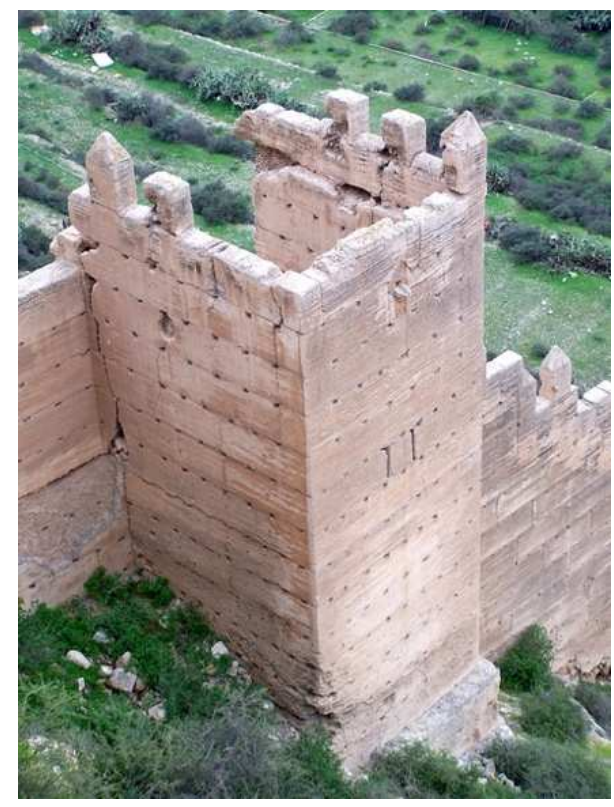

Fig. 6. Torre de flanqueo de la muralla de La Hoya (P. Gurriarán).

Cronológicamente, siempre se ha asociado estas obras con los reyezuelos taifas, gracias a las fuentes, especialmente con Jayrān al- 'Āmirí. La cita al empleo de tierra en esas construcciones servía para confirmar tal hipótesis, no obstante, recientes observaciones realizadas por Antonio Orihuela confirman que forran y engloban otra obra anterior de tapia casi desaparecida, tal vez esa muralla taifa. De este modo, su empleo se podría situar a comienzos del siglo XII, siempre tomando un término ante quem con la ejecución de las torres cristianas de mampostería.

\subsubsection{Tapias calicostradas de grano grueso}

Similares en su concepto tecnológico a los hormigones descritos antes, hay que hablar de la existencia de otras tapias calicostradas muy significativas en las fortificaciones almerienses. A diferencia de aquellas, la mezcla es más terrosa y parduzca, marcando claramente las tongadas, además de incluir en su masa mampuestos aislados pero organizados en distintos niveles. En su epidermis apenas se marcan las huellas de los encofrados.

Se reconocen con claridad estas fábricas en dos puntos tan significativos como la alcazaba o la muralla de San Cristóbal, generalmente asociadas a importantes reformas. Por ejemplo, en la ciudadela estas tapias sirven para levantar la mole del Mirador de la Odalisca, mientras que en el cerro vecino se usan para forrar las defensas al exterior, además de reconstruir dos torreones anteriores de tapia (Fig. 7). La hipótesis que defendemos desde hace tiempo es que se trataría de una fase constructiva emprendida por los almohades tras la toma de 1157 (Gurriarán, Márquez, 2005, p. 69).

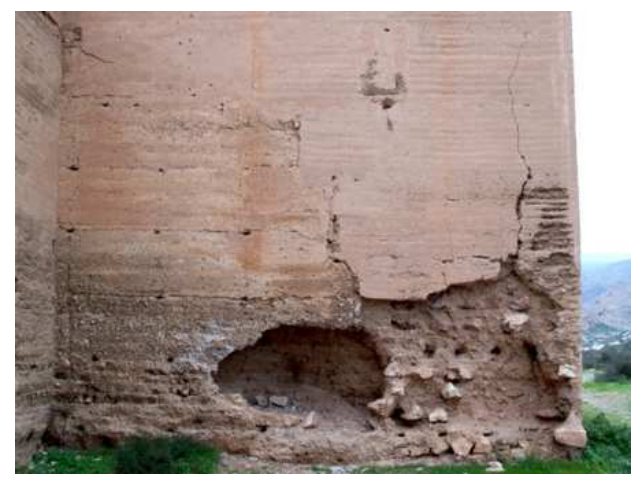

Fig. 7. Reparación de torre de flanqueo en la cerca del Cerro de San Cristóbal. Los restos originales (izq.) son calicostrados de grano fino, mientras que la nueva obra es calicostrada más gruesa (drch.) (P. Gurriarán).

\subsubsection{Tapias revestidas sobre zócalo de mam- postería}

Con esta denominación se hace referencia a unas tapias singulares que se observan en el frente norte de la alcazaba, especialmente en el primer recinto (Fig. 8). Estas fábricas se construyen con hormigones de cal de grano fino y curiosos mechinales en las esquinas de los cajones, generalmente de sección triangular. La longitud de cada encofrado no sobrepasa los dos metros. Apoyan sobre un potente zócalo de mampostería irregular, que se escalona para resolver los acusados desniveles de la peña. Al contrario que sucede en otros casos, aquí la mala calidad del material hizo que se aplicara un enlucido de cal de forma generalizada; sobre su superficie se pueden observar grandes esgrafiados con elementos geométricos (estrellas, retículas) y orgánicos (lágrimas). Estos lienzos se rematan con anchos merlones con albardillas piramidales, en 
cuyo seno aparecen pequeñas saeteras abiertas extrañamente hacia el exterior.

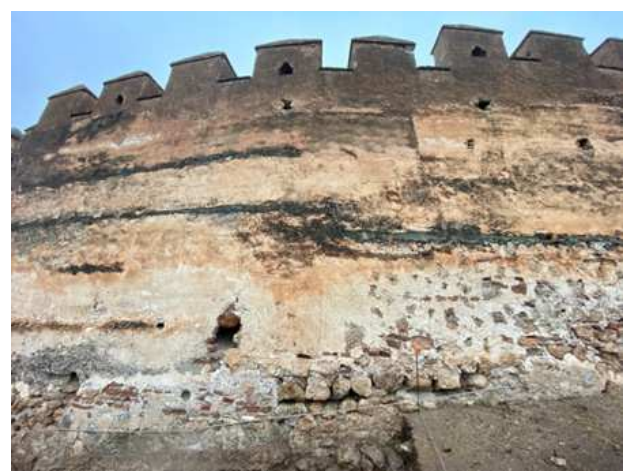

Fig. 8. Tapias nazaríes en el frente norte de la alcazaba (P. Gurriarán).

De acuerdo con la estratigrafía, se trata de una de las últimas fases andalusíes de la alcazaba. Un refuerzo de tiempos de los Reyes Católicos (post. 1490) que apoya contra uno de estos lienzos así lo demuestra. Lo precario de su obra y la comprobación de que amortiza otras tapias desmochadas y rotas, podría justificar su ejecución tal vez tras un terremoto.

\section{Conclusiones}

Este rápido repaso a algunas de las más significas técnicas constructivas de las murallas de Almería deja entrever su variedad y, sobre todo, la complejidad de su estudio. Hay que resaltar que cada obra o impulso constructivo responde a unas condiciones particulares, en las que diversos factores influyen en la conclusión de la obra arquitectónica: recursos económicos y técnicos, condiciones topográficas, urgencia, etc. La variedad identificada no solo refleja distintas épocas sino también la existencia de grupos de constructores o talleres con su propia especialización, generalmente establecidos en el lugar y, en muchos casos, durante largos periodos de tiempo como vemos en el caso de las tapias mixtas. Casos excepcionales como las torres de piedra de San Cristóbal, pueden definir la presencia de especialistas llegados desde otros lugares para ejecutar un proyecto concreto. En cualquier caso, la presencia de talleres de canteros en Almería entre mediados del siglo $\mathrm{X}$ y mediados del XI hubo de ser excepcional, y al igual que sucedió en otros puntos de al-Andalus, su trabajo caracterizado por las excepcionales sogas y tizones desapareció en beneficio de otros sistemas menos sofisticados. La mampostería y especialmente los tapiales acabaron siendo los principales recursos constructivos, y la variedad de tipologías identificadas de estos últimos, demuestra su versatilidad y depuración técnica a partir de similares materias primas. Las tapias, en definitiva, fueron las protagonistas en estas murallas, como sucede en muchos otros lugares de al-Andalus, sobre todo a partir del siglo XII.

\section{Notas}

Este trabajo se ha realizado dentro del marco del Proyecto de I+D del Subprograma Estatal de Generación del Conocimiento: "Las murallas medievales de Almería. Análisis cronotipológico y datación científica" (HAR2015-71609-P), cuyo IP es el Dr. Antonio Orihuela Uzal.

\section{Bibliography}

Acién Almansa, M. (2005). "La alcazaba de Almería durante los siglos IX-XI: cuestiones históricas y arqueológicas”, in Suárez Márquez, Á., coord., La Alcazaba. Fragmentos de una historia de Almería, Almería, pp. 75-87.

Al-Ḥimyarī (1938). La péninsule ibérique au Moyen Age d'après le Kitāb ar-Rawọ al-Mi tār, Lévi-Provençal, trad., E. Leiden.

Alcalá Lirio, F.; Arias de Haro, F. (2011). "Las viviendas de la Alcazaba”, in Monografías del Conjunto Monumental de la Alcazaba. Las últimas investigaciones en el Conjunto. Actas de las IV Jornadas Técnicas del Conjunto Monumental de la Alcazaba, Almería, tom. 3, pp. 61-83.

Alcaraz Hernández, F.M. (2002). "Excavación Arqueológica en Paseo de Almería, Puerta de Purchena y Rambla Obispo Orberá (Almería)", in Anuario Arqueológico de Andalucía. 1999, III. Actividades de Urgencia, Sevilla, vol. 1 , pp. 18-20. 
Arias de Haro, F. ; Alcalá Lirio, F. (2011). "El Palacio de la Alcazaba”, in Monografías del Conjunto Monumental de la Alcazaba. Las últimas investigaciones en el Conjunto. Actas de las IV Jornadas Técnicas del Conjunto Monumental de la Alcazaba, Almería, tom. 3, pp. 123-145.

Cara Barrionuevo, L.; Alonso Blanco, J.M.; Berbel Rodríguez, J. (2006). Expediente para la inscripción genérica de las canteras históricas de Almería, en el Catálogo General del Patrimonio Histórico Andaluz. Cantera y Canteras-cueva del Tesoro y de las Cuatro Cuevas, Almería, (inédito).

Castillo Galdeano, F.; Martínez Madrid, R. (1990). "La vivienda hispano-musulmana en Baŷȳāna-Pechina (Almería)”, in La casa hispano-musulmana. Aportaciones de la arqueología, Granada, pp. 111-127.

Gilotte, S., et al. (2010). "Los baños de la Tropa de la alcazaba de Almería: resultados preliminares de la intervención arqueológica”, Cuadernos de Madinat al-Zahra', 7, Córdoba, pp. 219-238.

Gurriarán Daza, P. ; Márquez Bueno, S. (2005). "La Almería medieval como fortaleza”, in Suárez Márquez, Á., coord., La Alcazaba. Fragmentos de una historia de Almería, Almería, pp. 57-72.

Gurriarán Daza, P. (2014). "Las técnicas constructivas en las fortificaciones andalusíes", in La ciutat medieval $i$ arqueologia. VI Curs Internacional d'Arqueologia Medieval, Lleida, pp. 263-292.

Ibn 'Id̄ārī. (1963). Al-Bayān al-mugrib, Nuevos fragmentos almorávides y almohades, Huici Miranda, A., trad., Valencia.

Lirola Delgado, J. (2005). Almería andalusí y su territorio. Textos geográficos, Almería.

Molina López, E. (2005). "Almería, ciudad mediterránea. Unas reflexiones, si cabe, oportunas”, in Monografías del Conjunto Monumental de la Alcazaba. Almería, Puerta del Mediterráneo (SS. X-XII). Actas de las II Jornadas Técnicas del Conjunto Monumental de la Alcazaba, Almería, tom. 1, pp. 11-24.

Navarro Luengo, I., et al. (1998). "Țurruš Jušayn y Munt Nīs: una propuesta de identificación para dos fortificaciones hafșūníes en la costa occidental malagueña", in Actas I Congreso Internacional Fortificaciones en alAndalus (Algeciras, 1996), Algeciras, pp. 433-439.

Pardo Barrionuevo, C.A. (2012). "Almería, de la Antigüedad a la Edad Media. La evolución urbana a través de la documentación arqueológica”, in Beltrán Fortes, J.; Rodríguez Gutiérrez, O., coord., Hispaniae urbes. Investigaciones arqueológicas en ciudades históricas, Sevilla, pp. 561-583. 
